\title{
Editorial
}

\section{Clinical photography and our responsibilities}

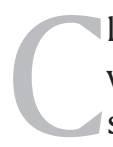

linical photographs in journals and textbooks are vital to illustrate a clinical finding, or an operative step or a postoperative result, thereby enriching and livening up the mundane words of text. Photographs are important for both medical record keeping and medical education, but have you ever stopped and pondered what does the patient get out of it? Is it not a one-way process in which the clinicians reap the benefit and the patient's interest are hardly of concern? Are we not treating the patients like an interesting case, an unusual finding, rather than living and feeling human being? Are we aware that an informed consent is required for clinical photography just like any other procedure?

Privacy of the patient and his/her health condition is nonnegotiable in the $21^{\text {st }}$ century. Health information is considered amongst the most sensitive and personal information that individuals possess. Just as patients must be able to trust their doctors with their lives and well-being, they should also be able to feel more than reassured that their health records are safe with the clinician. Besides caring for our patients, we must respect their dignity and privacy and protect their confidential information.

Photographs of patients are generally obtained within the doctor-patient relationship. This is particularly in Plastic Surgery where they form part of the patient's medical record, making them subject to this duty of confidence. Under any civil law, we are responsible for their confidentiality. Any confidential or personal information that is disclosed by a patient during consultation or obtained during clinical examination lies within the strict parameters of doctor/patient relationship, and can be used

\begin{tabular}{|l|l|}
\hline \multicolumn{2}{|c|}{ Access this article online } \\
\hline Quick Response Code: & Website: \\
\hline & www.ijps.org \\
\cline { 2 - 2 } & Dol: \\
\hline
\end{tabular}

only for the purpose for which it was disclosed, unless the patient agrees otherwise. Patients' photographic/ video records too are confidential documents and need to be respected similarly. Patients have autonomy over their bodies, and therefore, have the right to make their own decisions on medical treatment and allow subjecting themselves to photography/video filming. They also have the right to know for what purpose these will be used in future.

\section{USE OF CLINICAL PHOTOGRAPHS AND VIDEOS}

There are three principal uses of clinical photographs and videos - clinical, academic and research. Besides these, there is a very debatable fourth use which is commercial - where photographs and videos will be used as in advertisements.

\section{Clinical}

Photographs, videos and audios are an integral part of patient's clinical notes in Plastic Surgery and must be available throughout his/her follow-up period. Thereafter, they should be securely archived. In recent years, the paper documentation of medical records has given way to E-documentation. This has further increased the responsibility of the surgeon, as only he/she would be held responsible if the records are stolen and misused. Maintaining good clinical records is undoubtedly for the best interest of the patient but securing them from any misuse too remains our responsibility.

Photographs are an essential complement to a good clinical evaluation. Minor asymmetries can be picked up and highlighted. This is extremely important from a medico-legal aspect, to avoid allegations about improper or inadequate surgery. Accuracy in size, position, view, exposure, background, brightness and contrast are vital to derive conclusive results while comparing preoperative and postoperative photos 


\section{Academic}

The value of using photographs and video recordings of real patients to train medical students and para-medical staff is well-established. Although consent for this is not always obtained prior to taking the photos, standard practice is to obtain consent from the patient, or surrogate, prior to the dissemination of these modalities. There are several logical justifications for the use of patient videos in Plastic Surgery training:

1. There is a clear educational benefit of multimedia educational presentations in didactic lectures and seminars and of retrospective video quality analysis to assess the postoperative functional dexterity of a repaired palate or brachial plexus.

2. There is a benefit to the trainees and thereby the medical community, and to society, by improving the quality of care. Only three decades ago, microsurgery was being performed in select few centres but when the operative techniques of vessel and nerve anastomosis were seen in the department video libraries many trainees were inspired and today free tissue transfer is performed in even rural set ups!

3. Many teaching units may not be doing craniofacial surgery, aesthetic breast surgery and laser therapy, but training of residents can be augmented by video sessions conducted by visiting faculty.

The proposed audience includes trainees and health care professionals, who routinely encounter such settings and these videos/photographs/audio records present in the departmental archives are not to be made available to anyone else but genuine trainees and visiting trainees.

\section{Research}

Thesis, dissertations, publications all require photographs/ videos/audio clips to substantiate their research value. Now these are documents, which can slip into the public domain through open access journals, and patient's confidentiality becomes vital here.

\section{Commercial purposes}

The nonmedical use of recorded images for advertisement or commercial purposes can be an issue-morally, ethically and procedurally. In the same line, and perhaps a few steps behind, is the use of patient photographs/videos in surgeon's/hospital's web sites or their clandestine uploading on to the internet chat groups. Although some argue that in this there is an educational value to society, the primary motivations for filming, in many instances, are public education and financial benefit to proprietors.
Hence, instead of using photos of real patients for commercial purposes, one might argue that photographs of professional models should be used. But this conflicts against showing prospective patients true results of surgery. Moreover, one might be able to acquire before or after photos of a model, but obtaining both would be rare, and the model would also be a patient if they had undergone surgery! Furthermore, if the personal and hospital websites are making patients aware and reassuring them about the knowledge and competence level of a surgeon, whom they are about to choose, there is no guarantee that the surgeon will show honest results or even those of his own! So, is the sense of reassurance to the patient not misplaced?

There are numerous ethical arguments against the use of recorded images of patients for commercial purposes including:

1. Many patients are unable to consent prior to recording, due to the medical condition and emotional distress.

2. Patients who are technically able to consent may feel coerced in doing so, whether this is overt or camouflaged, in part due to the inherent vulnerability of their position as a patient.

3. Poverty in the developing world compels patients to attend busy public hospitals and teaching hospitals as they cannot afford care elsewhere, and are thus more vulnerable to video exploitation and the coercive forces of provider power imbalance and modern technology.

4. This act of denying individual autonomy to the patient for the common educational good of the masses threatens both rights of individuals to selfdetermination and society's trust in the medical profession.

5. While doctors taking their own photographs/videos may not be contested by the patient, the presence of a professional photography crew with all the technological gadgetry may be very embarrassing to the patient. This may also pose a significant distraction and conflict of interest, for already overburdened and stressed health care providers and ancillary hospital staff. A contrarian view is also prevalent, particularly in western countries-when one uses the services of the medical photography department, then everything is done in a formal professional way and the patient clearly understands that the photos are for their medical records. These patients may actually feel embarrassed to have photos taken on a surgeon's personal camera and wonder what they are going 
to be used for? Cultural perception and respecting patient's will is, therefore, vital.

6. Photographing/videographing patients without any tangible patient-centred benefit clearly violate both the letter and the spirit of these codes.

\section{SO WHAT IS EXPECTED OF US?}

Most patients are, usually, willing to have their pictures presented in conferences where they are assured that only doctors shall be attending, or for publication of their pictures in scientific journals, which doctors would be reading. The same patients may not be willing to have these photographs advertised in public magazines, newspapers or in posers at the surgeon's clinics. This sort of a partial consent should be specifically obtained as it helps the surgeon publish the permitted pictures for research, and also helps maintain privacy from the public eye for the patient. Archives on computers should be maintained under two broad categories-one where consent to public display of pictures has been obtained, and other where the patients have not consented to the same.

\section{Ideal practice of clinical photography would be}

1. Informed consent for the use of each image and each purpose must always be obtained from the patient or guardian.

2. Specific and fully informed consent for photography should always be sought and granted before taking photographs.

3. This consent may be withdrawn at any time.

4. Photography by a doctor/bonafide trainee/trained medical photographer in a suitable environment is the best.

5. During the photo session, the rights and dignity of the patient should always be respected.

6. All images must be stored in a safe and regulated environment, with controlled access.

7. Complete anonymity is impossible, but minimum possible area of the body should be photographed. Mere boxes or dots or shades over eyes do not make a photograph anonymous. Eyes should only be included when absolutely essential. Recognizable tattoos and birthmarks should be avoided in the frame, if they are not the reason for surgical intervention. Identifiable jewellery, hospital name tags should also be kept out of frame.

Images should generally not be obtained without specific prior consent. Patients should be asked about and consent to have images recorded and should be reassured that they may elect to decline participation or withdraw consent at any time. As with clinical procedures, for images taken for research purposes, consent should be informed and obtained carefully and with recognition that patients are a potentially vulnerable population that may feel overtly or implicitly coerced. Patients must be explicitly informed that refusing to have an image recorded will not affect their care but make follow-up difficult. If however, the surgeon feels uncomfortable with the attitude of the patient and comes to a conclusion that without a preoperative visual record he/she may not be able to defend himself/herself in a possible litigation in future, he/she should politely refuse to treat the patient.

There may rarely be circumstances when prior informed consent is not feasible, for example, filming of trauma resuscitations for quality improvement education. Such circumstances occur rarely, and patient confidentiality must be carefully protected. Subsequent use of such images should be limited to medical record documentation, or educational settings. Other uses require informed consent. Once the patient is stabilised, consent for the images taken should be obtained retrospectively, however, if the patient refuses consent, the images should be deleted.

Consent to disseminate the image should be obtained as a distinct question to the patient and should not be implied in the request to record the image. Patients must be properly informed of how the image may be disseminated, the purpose of the dissemination and the intended audience, before consent is requested.

\section{Images not revealing patient identity}

Many images taken from patients have a high educational value without revealing the patient's identity. X-rays, endoscopic images, images of specimens or tissues taken during the operation, still or video recordings of surgical procedures and microscopic images can all be useful teaching aids. Although they are derived from real patients, they can be classified as anonymous as they do not directly or indirectly, identify the person to whom they relate. These images are made fully anonymous when patient identifying details are erased/omitted from $\mathrm{X}$-rays and computed tomography/magnetic resonance scans.

If a patient is recognized in a case study, what are the harms that may occur? The main harm is the experience 
the patient has of violation of privacy that comes from having his/her information that was given in confidence, disclosed in the public arena. There is however a flaw in this reasoning. If we think that the only harm from a breach of confidentiality is experience of violation of the privacy of specific individuals, then no harm would be done if the person never found out, either through chance or because they are not capable of knowing. This would offer no protection to the illiterate, ignorant and the deceased. Hence wherever practicable, it is good practice, to obtain the patient's consent before either taking or using an image of them. Before using an image for educational purposes, all accompanying personal data should be removed. Ideally, it should be deleted completely so that the identity of the patient can never be re-established. Where there is a compelling reason for maintaining some method of identifying the patient, it should be done by way of a nonobvious code, key to which is stored securely in a different location from the image. Access to that key should be restricted as far as possible.

There is also a trend of developing online video libraries by various associations and societies and videographing patients in operative workshops. Both involve serious violation of patients' privacy and require a detailed and explicit consent from the patient. Any video procedure requires a special consent, even if the identity is not clearly visible. It should be made clear that the person taking the video is qualified, and that in no way it is a distraction to the surgeon. It is also a good idea to mention that steps will not be redone unnecessarily, just because it was not recorded properly the first time. One must be very cautious if these videos and libraries are being used for commercial purposes and double check if the patient has consented for the same. Taking snapshots and videos of the images projected on the screen from the auditorium has become a menace because this not only threatens the privacy of the patient but also intellectual property rights of the presenter/operating surgeon.

Many questions still remain unanswered. At what age are children old enough to understand the consequences and legally consent for photography? If a child is too shy to say no, or is admonished by strict parents to behave, is it ethical to photograph that child? It is a standard practice at some international conferences to copy all slides used, often without the consent of the presenter. How do we ensure that these copies do not fall in wrong hands and end up in undesirable web sites? It is desirable to watermark photographs, especially when sending across the net. The watermark ensures that it cannot be plagiarised. If someone does use it, that itself is illegal and open to legal redress.

There are many grey zones in this business of clinical photography, but once we have obtained consent for clicking, it becomes our responsibility to record, store, present, publish and distribute these photographic records responsibly, always keeping the interest of the patient topmost in our minds.

\section{Surajit Bhattacharya}

Editor

Indian Journal of Plastic Surgery

E-mail: surajitbh@yahoo.co.in

How to cite this article: Bhattacharya S. Clinical photography and our responsibilities. Indian J Plast Surg 2014;47:277-80..

Announcement

iPad App

Indian Journal of Plastic Surgery (IJPS) launches a dynamic app which optimizes the best in digital technology to enhance a print- like reading experience with multimedia links, videos and more.

- View abstracts, read full text, browse and get engaged in multimedia

- Complete content of each issue enhanced with iPad functionality

- Customized functions like search within an article and across the downloaded issues; highlight text and mark article as favorite

- Receive new issue notifications; convenient notification when a new issue is available

How to launch the app?

- Use the QR code

- Visit the App Store on your iPad and search for IJPS

- Download it from https://itunes.apple.com/in/app/indian-journal-plastic-surgery/id726088047 